\title{
Role of HCN4 channel in preventing ventricular arrhythmia
}

\author{
Kazuo Ueda ${ }^{1,6}$, Yuji Hirano ${ }^{2,6}$, Yasushi Higashiuesato ${ }^{3}$, Yoshiyasu Aizawa ${ }^{1,2}$, Takeharu Hayashi ${ }^{1}$, \\ Natsuko Inagaki ${ }^{1}$, Takeshi Tana ${ }^{3}$, Yusuke Ohya ${ }^{3}$, Shuichi Takishita ${ }^{3}$, Hiromi Muratani ${ }^{3,4}$, Masayasu Hiraoka ${ }^{2}$ \\ and Akinori Kimura ${ }^{1,5}$
}

Bradycardia is a trigger of ventricular arrhythmias in patients with arrhythmia including Brugada syndrome and long QT syndrome. The HCN4 channel controls the heart rate, and its mutations predispose to inherited sick sinus syndrome and long QT syndrome associated with bradycardia. We found a 4 base-insertion at the splice donor site of the HCN4 gene in a patient with idiopathic ventricular tachycardia, which was supposed to generate a truncated channel. To investigate the role of the HCN4 channel in ventricular arrhythmia, we introduced a ventricular action potential of $l_{\mathrm{f}}$ channel produced by HCN4 in a computer simulation model and found that the $I_{\mathrm{f}}$ channel generated a leaky outward current during the plateau phase of ventricular action potential. Currents through the $I_{\mathrm{f}}$ channel were suggested to contribute to the shortening of the action potential duration and the prevention of early after-depolarization in bradycardia. These observations suggested that the HCN4 channel played a preventive role in triggering bradycardia-induced ventricular arrhythmias.

Journal of Human Genetics (2009) 54, 115-121; doi:10.1038/jhg.2008.16; published online 23 January 2009

Keywords: bradycardia; computer simulation; HCN4; mutation; ventricular arrhythmias

\section{INTRODUCTION}

Sinus node dysfunction (SND) is characterized by the slow heart rate accompanied by rhythm disturbance. Patients with SND usually show a benign clinical course, but some patients with severe dysfunction require an artificial pacemaker implantation. Most cardiac arrhythmias are associated with acquired pathological conditions of the heart, including ischemic heart disease, hypertensive heart disease and congestive heart failure. Mutations in genes for cardiac ion channels are known to cause $\mathrm{SND}^{1,2}$ and other arrhythmias, including long QT syndrome (LQTS), ${ }^{1,3-9}$ short QT syndrome, ${ }^{10,11}$ familial atrial fibrillation, ${ }^{12,13}$ Brugada syndrome $(\mathrm{BrS}),{ }^{14}$ progressive cardiac conduction disturbance $^{15}$ and polymorphic ventricular tachycardia (PVT). ${ }^{16}$ Functional alterations caused by the channel gene mutations have clarified the role of each channel in cardiac excitation and conduction in normal and abnormal conditions. ${ }^{17}$

We earlier reported a mutation of gene for hyperpolarizationactivated channel 4 (HCN4 channel or $I_{\mathrm{f}}$ channel) in a patient suffering from SND, who also showed QT prolongation in ECG and developed PVT. ${ }^{1}$ The $I_{\mathrm{f}}$ channel is preferentially expressed in cells with automaticity controlling the heart rate, and it is called as a pacemaker channel. A functional study of the mutation showed a severe loss of function of HCN4 channel, which may explain the SND phenotype. The $I_{\mathrm{f}}$ channel is also expressed in the working myocardium, ${ }^{1,18}$ raising a question whether there is another role in the working myocardium other than that involved in the automaticity of atrioventricular node and His-Purkinje cells. The PVT observed in the patient with the mutation implied that the $I_{\mathrm{f}}$ channel dysfunction might be associated with provoking ventricular arrhythmia.

It is well known that bradycardia (lower heart rate) is associated with a higher incidence of sudden death during sleep in LQTS type 3 (LQTS3) and BrS. LQTS3 and about $30 \%$ of BrS are caused by SCN5A mutations, and compound heterozygotes of SCN5A mutations were reported to express SND, suggesting that the $S C N 5 A$-associated SND was a recessive disorder. On the other hand, in the majority of BrS patients who do not carry the SCN5A mutations, it remains unknown which genetic factor confers a risk of bradycardia-induced ventricular arrhythmias. Concerning the LQTS, it was reported that double mutation cases of two arrhythmia-associated genes were $7.9 \%$, relatively common than formerly expected, and that cumulative effects of disease-related genes would increase the risk of arrhythmia in severe cases. ${ }^{19}$

\footnotetext{
${ }^{1}$ Department of Molecular Pathogenesis, Tokyo Medical and Dental University, Tokyo, Japan; ${ }^{2}$ Division of Pathophysiology, Medical Research Institute, Tokyo Medical and Dental University, Tokyo, Japan; ${ }^{3}$ Third Department of Internal Medicine, University of the Ryukyus, Okinawa, Japan; ${ }^{4}$ Center for Health and Sports Science, Kyushu Sangyo University, Fukuoka, Japan and ${ }^{5}$ Laboratory of Genome Diversity, School of Biomedical Science, Tokyo Medical and Dental University, Tokyo, Japan

Correspondence: Professor A Kimura, Department of Molecular Pathogenesis, Medical Research Institute, Tokyo Medical and Dental University, 1-5-45 Yushima, Bunkyo-ku, Tokyo 113-8510, Japan.

E-mail: akitis@tmd.ac.jp

${ }^{6}$ These authors contributed equally to this work.

Received 12 November 2008; revised 9 December 2008; accepted 19 December 2008; published online 23 January 2009
} 
In this study, we searched for mutations in the HCN4 channel gene ( HCN4) in patients with ventricular arrhythmia, analysed the functional alteration caused by the mutation and investigated how the pacemaker channel was involved in the bradycardia-induced ventricular arrhythmia by using a simulation model.

\section{MATERIALS AND METHODS}

\section{Subjects}

Fifty-four and thirty-three genetically unrelated patients with LQTS and BrS, respectively, were the subjects in this study. Control subjects were 190 genetically unrelated healthy individuals. A blood sample was obtained from each subject after informed consent was obtained. Genomic DNAs were extracted from the blood samples and analysed for mutations in the known disease genes for cardiac arrhythmia, including KCNQ1, KCNH2, SCN5A, KCNE1, KCNE2 and KCNJ2 by PCR and single-strand conformational polymorphism (SSCP) method, ${ }^{20}$ using primers reported in the literatures. ${ }^{7,21-23}$ The research protocol was approved by the Ethics Reviewing Committee of Medical Research Institute, Tokyo Medical and Dental University.

\section{Mutational screening in $\mathrm{HCN} 4$}

Exons of HCN4 were separately amplified using pairs of primers (Table 1). The PCR products from patients were searched for sequence variations by the PCRSSCP method ${ }^{20}$ and subsequent sequencing of PCR products cloned into pCRII vectors (Invitrogen, Carlsbad, CA, USA). Several clones were sequenced to ensure that the sequence variation was not the PCR artefacts.

\section{Functional analysis of a splice site mutation}

To investigate the functional changes caused by the splicing donor site mutation, exon 2 and its flanking intron sequences of HCN4 were amplified by PCR with primers ET2-F (5'-GAATTCTCTGTAACTTGTCCTCTCTC-3') and either ET2-WR (5'-GATATCTTACCTCTTCCCACTGGTG-3') or ET2-MR (5'-GATATCTTACTCACCTCTTCCCACT- $\left.{ }^{\prime}\right)$ from the patient carrying the mutation. The PCR products were cloned into pSPL3 at EcoRI and EcoRV sites. Several independent clones were isolated and sequenced to ensure that the fragment was free from errors in PCR. COS7 cells were cultured in Dulbecco's modified Eagle medium supplemented with $10 \%$ fetal calf serum (GIBCO BRL) and antibiotics. The cells were transfected with either wild type or mutant construct $(2 \mu \mathrm{g}$ per $10 \mathrm{~cm}$ dish) using SuperfectAMINE (Qiagen Japan, Tokyo, Japan) according to the manufacturer's instructions. Total mRNA from the transfected cells was extracted and used for generating cDNA templates. SSCP analysis was performed on the reverse transcription (RT)-PCR products amplified with an EX2S primer (5'-CGATATATTCACCAGTGGGAA-3') and a VA primer (5'-CACCTTCTGATAGGCAGCCT- $3^{\prime}$ ); the former primer corresponded to exon 2 of HCN4 and the latter primer was corresponded to the pSPL3-encoded exon. An aberrant splicing was detected by using sequencespecific primers. When the natural splice donor site was used, the NS (5'-TTCACCAGTGGGAAGAGACC- ${ }^{\prime}$ ) and VA primers would generate a PCR product. On the other hand, when the mutant sequence could work as a splice donor, the aberrant splicing product should be amplified by the IS (5'-CCAGTGGGAAGAGGTGAACC- $3^{\prime}$ ) and VA primers. The sequencespecific primers, NS and IS, span the junction of the HCN4 exon and the vector-derived exon.

\section{Electrophysiological analysis of HCN4 channel}

COS7 cells were co-transfected with $0.4 \mu \mathrm{g}$ pEGFP-C1 (Clontech, Mountain View, CA, USA) and $1.6 \mu \mathrm{g}$ rabbit HCN4 cDNA (GenBank Accession no. AB022927) cloned into PCI (Promega, Madison, WI, USA). The transfected cells were removed from the $35 \mathrm{~mm}$ culture dish $36-48 \mathrm{~h}$ after transfection and placed into a chamber on the stage of an inverted microscope (Diaphoto TMD, Nikon). Thereafter, currents were recorded by a whole-cell patch-clamp configuration using an amplifier (Axopatch 200B, Axon Instrument) from cells expressing co-transfected pEGFP-C1, as described previously. ${ }^{1}$ After forming the whole cell configuration, cell membrane capacitance was estimated by analysing the capacity transient elicited by $5 \mathrm{mV}$ hyperpolarizing pulses. Series resistance compensation was done by $50-70 \%$ using the circuit built into an amplifier (Axopatch 200B, Axon Instruments, Foster City, CA USA). The voltagedependence of tail currents was analysed by fitting to the Boltzmann equation of

$$
g / g_{\max }=\left(1-\min P_{0}\right) /\left[1+\exp \left\{\left(V-V_{1 / 2}\right) / \kappa\right\}\right]+\min P_{0}
$$

according to the method of Chen $\mathrm{J}$ et al., ${ }^{24}$ where $V_{1 / 2}, \kappa$ and $\min P_{0}$ are half maximal activation voltage, slope factor and the minimum open probability (defined as the minimum value of relative tail current), respectively. Software (pCLAMP8, Axon Instruments) was used to generate voltage protocols, to acquire data and to analyse current signals. All experiments were done at $34 \pm 1.0^{\circ} \mathrm{C}$. Data were obtained from eight cells and represented by mean \pm s.e.m.

Computer simulation of $I_{\mathrm{f}}$ current on ventricular action potential On the basis of our experimental data, we implemented the current through the $I_{\mathrm{f}}$ channel into action potential models, including two human ventricular

Table 1 PCR primers used in the analysis of genomic gene for human $\mathrm{HCN} 4^{\mathrm{a}}\left(5^{\prime} \rightarrow 3^{\prime}\right)$

\begin{tabular}{|c|c|c|c|}
\hline Exon & Forward primer & Reverse primer & PCR product (bp) \\
\hline 1.1 & TGGCGCCATGGACAAGCTGC & AAGCGCCTGCAGTCGCCGTT & 295 \\
\hline 1.2 & GCGGGCAAGTCCAGCACGAA & CGCGCCTCCCTCCACTTTGA & 334 \\
\hline 1.3 & AGCAGCCCTCGGTGGACA & ACCCACAGGATCATCGCTGT & 278 \\
\hline 2.1 & СTCTCTCTCTTCCTGGCGACT & TCCACGGGGATGGAGGAAAT & 316 \\
\hline 2.2 & GACCCGCAGCGGATTAAAAT & TTTCCCCCAAGAGGTTTGCA & 258 \\
\hline 3 & GCCACTCACCTATCCCAGCC & CCGCCTATGGCCCAGAGAGA & 248 \\
\hline 4 & СТTСССТСТСАТССАСТGТС & TTGGGAGGGACCAATGTGCG & 305 \\
\hline 5 & CTGGTGGGGTGGAGCTGA & GACAGGGTGGATTGGGACAC & 262 \\
\hline 6 & TCACСАССТССТTCCCGTGT & CAAACCAGCCCCTGGGAGCA & 339 \\
\hline 7 & ATGCCTCATCCTGAGTCCTGA & TAAAGGAGCCCTGCCCTCCT & 264 \\
\hline 8.1 & TTCCCCGCTCTGCCCTGAGT & AGGGTGGTGGGTGAGGGCTAT & 299 \\
\hline 8.2 & TCATCTGGACCCCGCTGATC & CAGAGAATCCAGCCAGCTGTT & 286 \\
\hline 8.3 & GATCCCTTCTGCGCTGGGCT & TGAGCAGGGGAGAGTCGGAG & 285 \\
\hline 8.4 & CCACCACCATAGCCGGGTTT & TAAAGCCTACAGGGGAAGCC & 358 \\
\hline 8.5 & CACTGAGCACGCCAGAGACA & GATGCAGGTGGCAGGAGCAA & 206 \\
\hline 8.6 & CCССССAAGAACCTTCCCGA & ATGGACTCCCCTGAGGAGT & 241 \\
\hline 8.7 & TCTCAGCCAGCCCTGCCTCA & CAGGTTCCCTCTGGGGTCCA & 302 \\
\hline 8.8 & CAAGAGCCACCTCTTCTGGG & GGTATATCTCCTAATCACAGTTAAACCT & 188 \\
\hline
\end{tabular}

aprimers used for the analysis of HCN4. Exons 1, 2 and 8 were analysed as three, two and eight overlapping fragments, respectively. 
action potential models (the Priebe-Beuckelmann model for heart failure ${ }^{25}$ and the Tusscher-Noble-Noble-Panfilov model ${ }^{26}$ ) and the Luo-Rudy model ${ }^{27}$ that was originally proposed for guinea-pig ventricular myocytes. $I_{\mathrm{f}}$ was formulated by a classical Hodgkin-Huxley-type gating scheme, using the variable for activation gate, $y$,

$$
\begin{aligned}
& I_{\mathrm{f}}=g_{\mathrm{f}, \mathrm{Na}} \times y \times\left(V-E_{\mathrm{Na}}\right)+g_{\mathrm{f}, \mathrm{K}} \times y \times\left(V-E_{\mathrm{K}}\right) \\
& \mathrm{d} y / \mathrm{d} t=\left(y_{\text {inf }}-y\right) / \tau
\end{aligned}
$$

in which $g_{\mathrm{f}, \mathrm{Na}}$ and $g_{\mathrm{f}, \mathrm{K}}$ represent the conductance of $\mathrm{Na}^{+}$and $\mathrm{K}^{+}$components of $I_{\mathrm{f}}$, respectively, and $E_{\mathrm{Na}}$ and $E_{\mathrm{K}}$ represent equilibrium potentials for $\mathrm{Na}^{+}$and $\mathrm{K}^{+}$. The time constant $(\tau)$ and activation curve $\left(y_{\text {inf }}\right)$ obtained in this study were used for the implementation of the $I_{\mathrm{f}}$ current. The value of $g_{\mathrm{f}, \mathrm{Na}}$ and $g_{\mathrm{f}, \mathrm{K}}$, and the current density of $I_{\mathrm{f}}$ were from a report analysing human ventricular myocytes. ${ }^{28} g_{\mathrm{f}, \mathrm{Na}}$ was set to $0.0032 \mathrm{mS}_{\mu} \mathrm{F}^{-1}$ and $g_{\mathrm{f}, \mathrm{K}}$ to $0.011 \mathrm{mS}_{\mu} \mathrm{F}^{-1}$, consistent with a value of $P_{\mathrm{K}} / P_{\mathrm{Na}}=3.3$ determined by the reversal potential measurement in this study. The outcome of $I_{\mathrm{f}}$ incorporation was qualitatively similar in different action potential models.

\section{RESULTS}

Identification of HCN4 mutation in patients with ventricular arrhythmia

To identify arrhythmia-related mutations in patients with idiopathic ventricular arrhythmia, we analysed 54 LQTS patients and $33 \mathrm{BrS}$ patients for mutations in the known arrhythmia-causing genes, including KCNQ1, KCNH2, SCN5A, KCNE1, KCNE2, and KCNJ2. We identified Gly229Asn (G-to-A transition at position 686) and Phe275del (3 nucleotides deletion at position 824-826) as LQTSassociated KCNQ1 mutations. In addition, Phe627Ile (T-to-A transition at position 1879), Phe640del (3 nucleotides deletion at positions 1918-1920), Leu987fs (2 nucleotides deletion at positions 2959 and 2960), Arg1033fs (C insertion at position 3098), Val1074fs (T deletion at position 3221) were found in $\mathrm{KCNH} 2$ from five different LQTS patients. A Gly146Ser (G-to-A transition at position 436) was found in KCNJ2 from an LQTS patient with periodic paralysis (LQTS7 phenotype). Regarding SCN5A, Met1296fs (T insertion at position 3888) was found in a BrS patient. These mutations were not found in 380 control chromosomes and no compound heterozygotes of mutations were found in this study.

We next investigated for HCN4 mutations in the patients. The analysis revealed an HCN4 mutation in heterozygous state in a symptomatic idiopathic ventricular tachycardia patient who had no SCN5A mutation. This mutation, IVS2DS, was an insertion of four bases at a splicing junction of exon 2 and intron 2 (Figure $1 \mathrm{~b}$ ), which was not found in the controls. The patient with IVS2DS, a 41-year-old man who suffered from recurrent episodes of syncope at rest, was admitted to a hospital, and his ECG showed typical ST-elevation in V1-2 (saddle-back type as shown in V2, Figure 1c) and incomplete right bundle branch block. The QT intervals corrected by the heart rate, QTc, were between 0.450 and $0.475 \mathrm{~s}$, prolonged to an upper limit of normal range. His 24-h ECG recording showed PVT during sleep. An electrophysiological study showed that ventricular fibrillation was reproducibly induced by programmed ventricular stimulation without any drug provocation. From the clinical manifestation and response to electrophysiological study, he was diagnosed as having BrS and was implanted with an implantable cardioverter defibrillator. He had no apparent family history of sudden cardiac death, but genetic analysis of his family members could not be carried out because they refused genetic testing.

\section{Functional analysis of IVS2DS}

As the four-base insertion was found at the splicing donor site, Inserted bases might be expressed as a part of mRNA (Figure 1b).
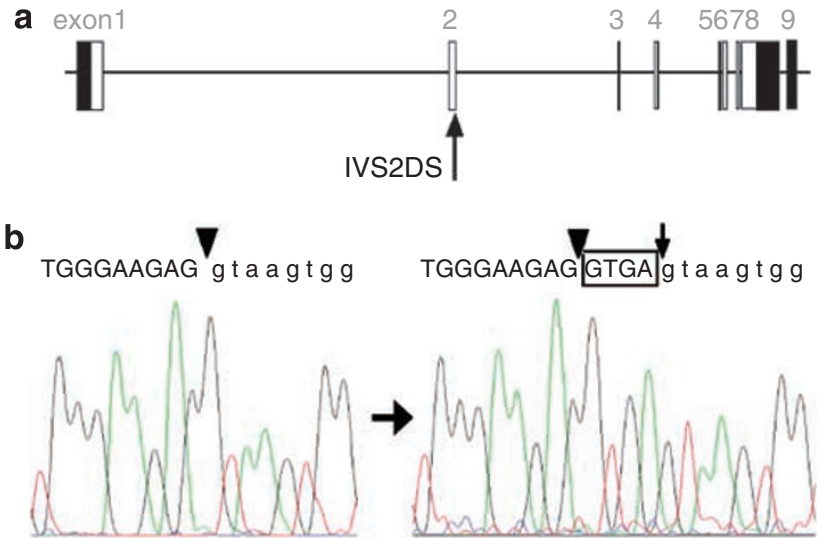

C

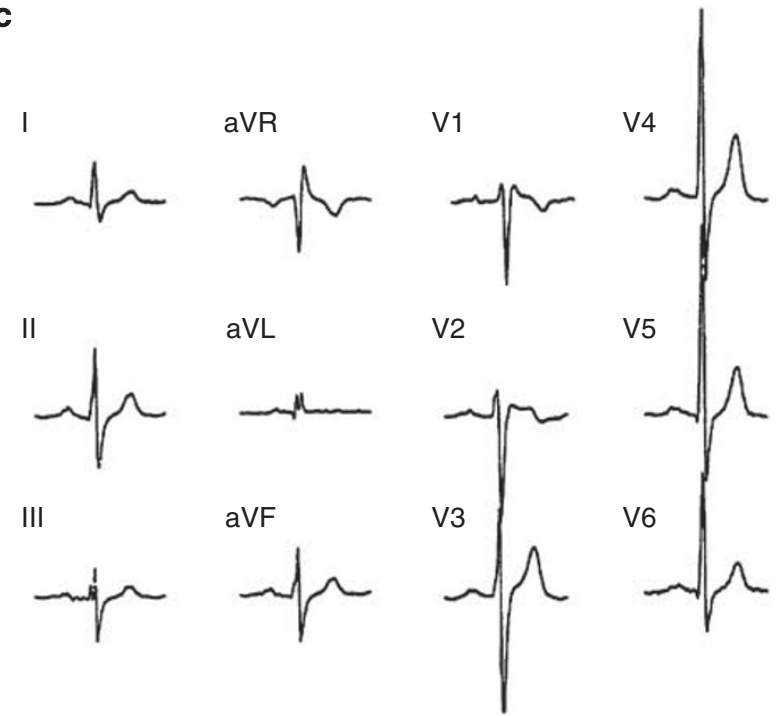

Figure 1 Genetic analysis of patients with idiopathic arrhythmia for $\mathrm{HCN} 4$ mutation. (a) The variant, IVS2DS, is represented on the genomic structure of HCN4. (b) Sequence profiles of plasmid clones containing normal and IVS2DS-derived exon 2 and intron 2. IVS2DS was an insertion of four nucleotides (enclosed within square) at the splicing donor site. An arrowhead and an arrow indicate a normal exon-intron boundary and a putative splicing position in the variant allele, respectively. (c) ECG of patient with the HCN4 mutation showed typical ST-elevation in V1-2 and incomplete right bundle branch block.

When the insertion was transcribed as a part of mRNA, it was predicted to cause a frameshift with an Ile-to-Val change at codon 404 and following non-relevant 43 amino acids, that is, HCN4 carrying IVS2DS might encode a truncated HCN4 channel. As heart biopsy sample was not obtained from the patient, the possible functional alteration owing to the splice donor site mutation was investigated by using a transfection experiment with artificial minigene constructs. PCR fragments containing the splicing junction of wild type (WT) and variant (or insertion, IS) alleles from the patients were cloned into pSPL3 (Figure 2a) and transfected into COS7 cells to analyse the splicing products by the RT-PCR method. The IS construct was found to generate an additional RT-PCR product not present in cells transfected with the WT construct (Figure 2b), suggesting that the additional 4 bases after the natural splice donor site were incorporated into mRNA. The aberrant splicing was detected by the RT-PCR method using combinations of sequence-specific primers, as the insertion-specific RT-PCR product was found only in the cells transfected with the IS construct (Figure $2 \mathrm{c}$ ). The splice product using 

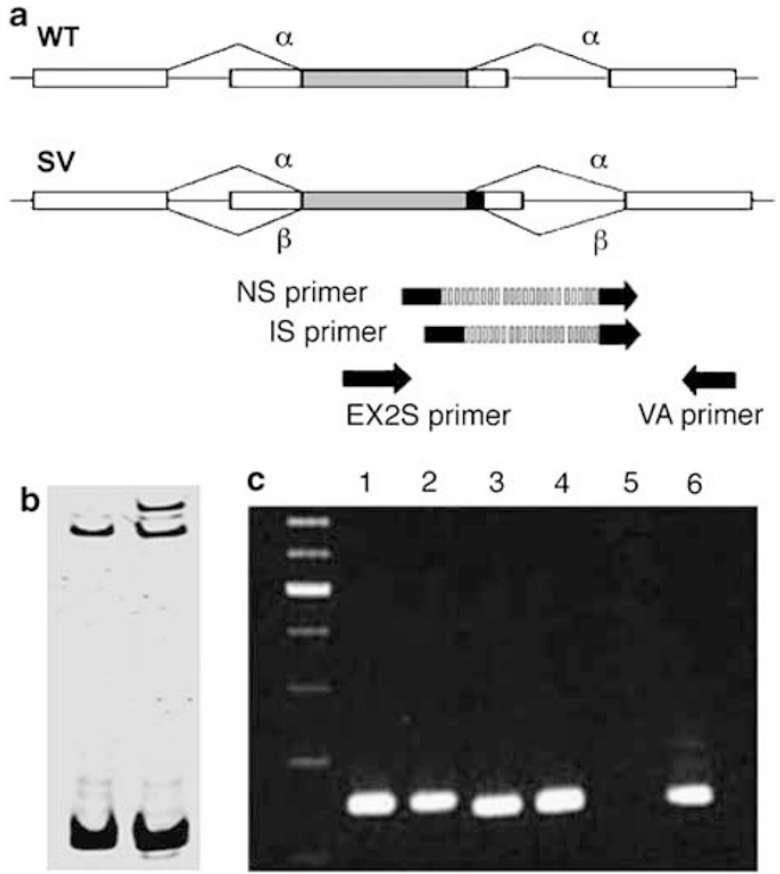

Figure 2 Splicing analysis of IVS2DS. (a) Schematic representations of wild type and variant constructs cloned into pSPL3. Exon 2 of HCN4 is represented by a shaded box and additional four nucleotides of GTGA in IVS2DS are indicated by a filled box. Normal splicing events are shown by $\alpha$, whereas a predicted aberrant splicing is denoted by $\beta$. Positions of primers used in the RT-PCR analysis are shown: EX2S,exon2-specific primer; NS, normal splicing-specific primer; IS, insertion-associated splicing-specific primer; and VA, vector-associated primer. (b) SSCP pattern of RT-PCR products obtained with EX2S and VA primers from COS7 cells transfected with either wild-type (left) or variant (right) constructs. An additional RT-PCR product (arrowhead) was detected only for the variant. (c) RT-PCR analysis to detect the aberrant splicing. Lanes 1,3 and 5 are derived from cells transfected with the WT construct, and lanes 2, 4 and 6 are derived from that with the variant construct. The primers used are EX2S and VA in lanes1 and 2 , NS and VA in lanes 3 and 4 , and IS and VA in lanes 5 and 6.

the natural donor site was also found with IS (Figure 2c), and the SSCP profile suggested that almost equal amount of normal and abnormal splicing products were expressed (Figure $2 b$ ).

\section{Electrophysiological analysis of HCN4 channel}

To explore the possible contribution of the $I_{\mathrm{f}}$ current to the action potential of ventricular cells, we used computer simulation studies. As there was no previous data for incorporating $I_{\mathrm{f}}$ to be used in the simulation study, we conducted the whole-cell patch-clamp analysis of COS7 cells transfected with a rabbit HCN4 construct to obtain the functional characteristics of the HCN4 channel. When hyperpolarizing test pulses were applied from a holding potential of $-30 \mathrm{mV}$, voltageand time-dependent inward currents were activated at potentials negative to $-60 \mathrm{mV}$ (Figures 3a and c), which were similar to the $I_{\mathrm{f}}$ current recorded in native ventricular cardiomyocytes except for the less negative activation voltage. ${ }^{28}$ Tail currents were obtained at membrane potential shifted to $-100 \mathrm{mV}$ after various test hyperpolarizations (Figure $3 \mathrm{~b}$ ). The time-course of current activation became faster (Figure $3 \mathrm{~d}$ ) with hyperpolarization of test pulses negative to $-60 \mathrm{mV}$. Voltage-dependence of $I_{\mathrm{f}}$ current was analysed by fitting to the Boltzmann equation to obtain various parameters of the HCN4 channel function; $V_{1 / 2}=-77.98 \pm 1.06, \kappa=9.11 \pm 1.03$, and $\min P_{0}$ was $0.109 \pm 0.029$. a
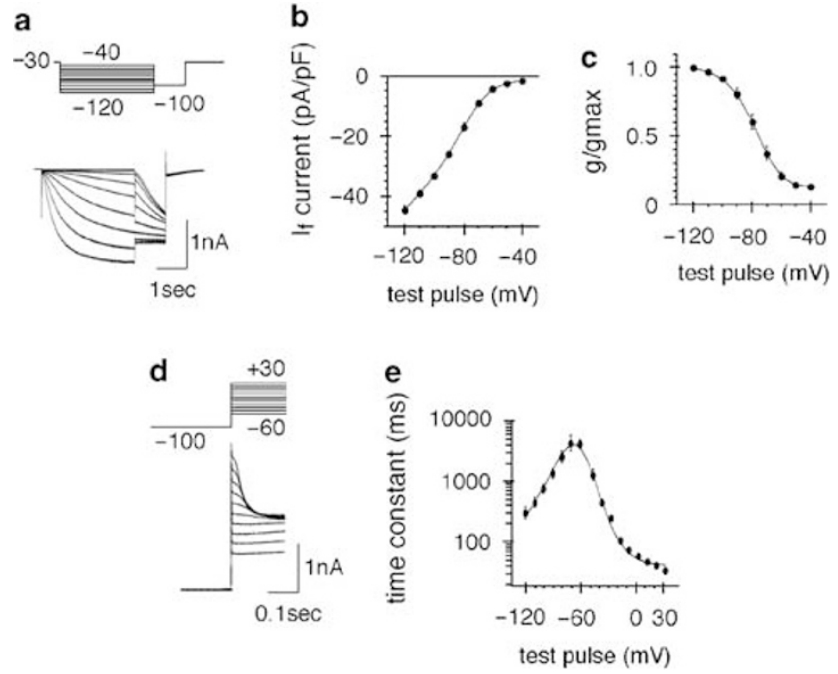

Figure 3 Electrophysiological properties of hyperpolarization-activated current $\left(I_{f}\right)$ generated by HCN4 channel. Representative records of HCN4 channel currents and data analysis are shown. (a) The $I_{f}$ current through HCN4 channel was activated during hyperpolarizing test pulses of $3 \mathrm{~s}$ between -40 and $-120 \mathrm{mV}$ in $10 \mathrm{mV}$ increments from a holding potential of $-30 \mathrm{mV}$, and tail currents were obtained at $-100 \mathrm{mV}$ after the test pulses. The data were analysed to show $I_{f}-V$ relationship curve (b) and normalized tail current- $V$ relationship curve (c). $V_{1 / 2}, \kappa$ and $\min P_{0}$ were $-77.98 \pm 1.06, \quad 9.11 \pm 1.03$ and $0.109 \pm 0.029$, respectively. (d) Deactivation currents were recorded during test voltages between -60 and $30 \mathrm{mV}$ in $10 \mathrm{mV}$ increments after the conditioning pulse to $-100 \mathrm{mV}$ from the holding potential of $-30 \mathrm{mV}$. The data for activation and deactivation currents were used for drawing a time constant curve in logarithmic scale (e). Absence of error bars indicates errors smaller than the symbol size.

Effects of $I_{\mathrm{f}}$ current on action potential in a simulation model

The $I_{\mathrm{f}}$ channels expressed in the heart are mainly composed of HCN2 and HCN4 channels. According to the previous reports, ${ }^{24,29}$ electrophysiological properties, $V_{1 / 2}$ and $\tau$, of HCN2 channel suggested that more HCN4 channel could be open than the HCN2 channel under the physiological condition, and $\min P_{0}$ of the HCN2 channel was similar to that obtained in our study. Thus, $V_{1 / 2}, \tau, \min P_{0}$ and time constant of HCN4 channel obtained in this study were used as parameters of the $I_{\mathrm{f}}$ channel in the simulation models. ${ }^{30}$

Figure 4 illustrates the effects of $I_{\mathrm{f}}$ current introduced into a human ventricular action model proposed by Tusscher et al. (TusscherNoble-Noble-Panfilov model). ${ }^{26}$ As shown in Figure 4a, incorporation of $I_{\mathrm{f}}$ current shortened the action potential duration (APD) and depolarized the resting membrane potential minimally. Being a hyperpolarization-activated current, $I_{\mathrm{f}}$ was activated during the diastole and deactivated following the upstroke of the action potential. This gating produced a decaying outward current at the plateau phase, as $I_{\mathrm{f}}$ had mixed the conductance for $\mathrm{Na}^{+}$and $\mathrm{K}^{+}$leading to a reversal potential around $-30 \mathrm{mV}$.

In the Tusscher-Noble-Noble-Panfilov model, the amplitude of $I_{\mathrm{f}}$ was comparable with other outward currents, including $I_{\mathrm{Kr}}$ and $I_{\mathrm{Ks}}$. It should be noted that the amplitude of $I_{\mathrm{f}}$ was stimulus-frequency dependent, as the degree of activation was determined during diastolic interval (Figure 4d). Hence, the extent of APD shortening was augmented when the basic cycle length (BCL) was increased. It was found that, with BCL of 1000, 2000 and $4000 \mathrm{~ms}$, inclusion of $I_{\mathrm{f}}$ current shortened the $\mathrm{APD}_{90}$ by $9.6 \mathrm{~ms} \quad(3.5 \%$ of the control $\left.\mathrm{APD}_{90}\right), 10.2 \mathrm{~ms}(3.7 \%)$ and $10.6 \mathrm{~ms}(3.8 \%)$, respectively. The APD shortening by the incorporation of $I_{\mathrm{f}}$ was a consistent finding in all 

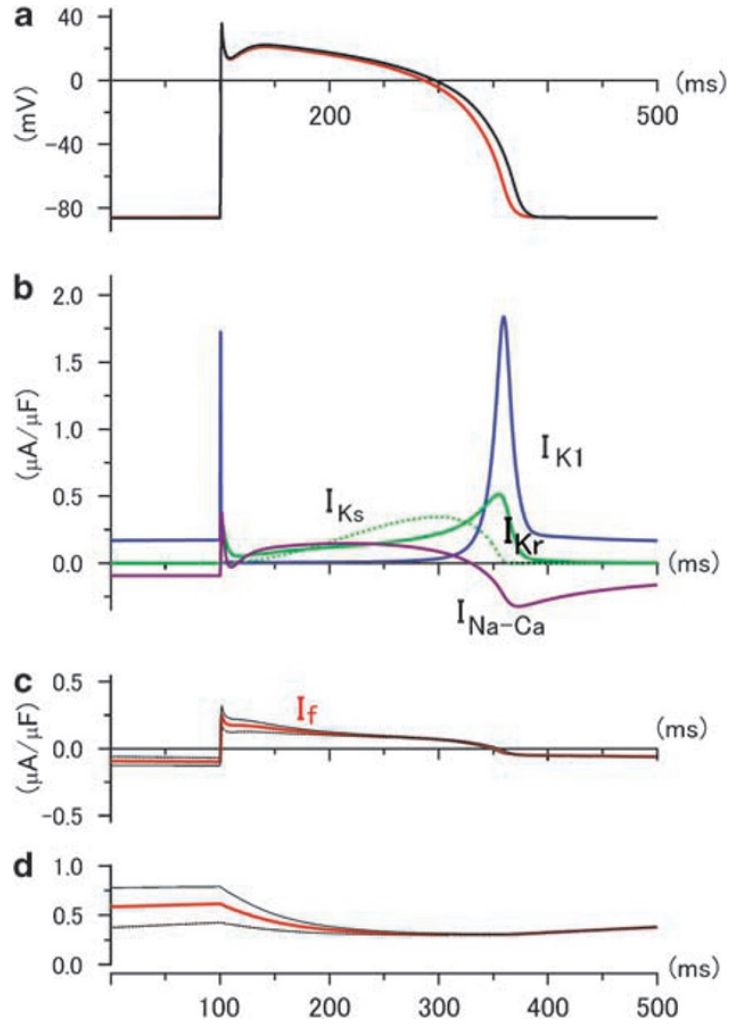

Figure 4 Ventricular action potential model incorporating the $I_{f}$ current. Properties of the $I_{f}$ currents were incorporated into the TNNP human ventricular action potential model based on the results shown in Figure 3. (a) Action potentials with and without $I_{f}$ incorporation were shown with red and black lines, respectively. Models were paced with a basic cycle length (BCL) of $1000 \mathrm{~ms}$. (b) Temporal profiles of current systems during the action potential with $I_{\mathrm{f}}$ incorporation. Inward rectifier $K$ current $\left(I_{K 1}\right)$ : blue; delayed rectifier $K$ currents $\left(I_{\mathrm{Ks}}\right.$ and $\left.I_{\mathrm{Kr}}\right)$ : green; $\mathrm{Na}-\mathrm{Ca}$ exchange current $\left(/_{\mathrm{Na}-\mathrm{Ca}}\right)$ : purple. (c) Temporal profiles of $I_{f}$ current (red line). Current amplitudes with BCL of $500 \mathrm{~ms}$ (dotted black line) and $2000 \mathrm{~ms}$ (thin black line) were superposed. (d) Values of activation gate (red line). Values with BCL of $500 \mathrm{~ms}$ (dotted black line) and $2000 \mathrm{~ms}$ (thin black line) were superposed.

action potential models, whereas the extent of shortening showed variations. When $I_{\mathrm{f}}$ was introduced into the Luo-Rudy model, ${ }^{27}$ decrease in $\mathrm{APD}_{90}$ was $1.9 \mathrm{~ms}$ or $1 \%$ of the control value when $\mathrm{BCL}$ was set to $1000 \mathrm{~ms}$.

\section{Effects of $I_{\mathrm{f}}$ current on action potentials under pathological conditions}

Triggering of arrhythmias in the inherited arrhythmogenic diseases generally requires particular situations, such as physical or emotional stress and additional APD prolongation associated with specific medications or bradycardia. The underlying mechanisms of these events may be analogous, for example, with the onset of lethal arrhythmias in patients with cardiac failure or myocardial ischemia. Therefore, we were concerned with the effects of $I_{\mathrm{f}}$ current when cardiomyocytes were under pathological or unusual situations.

Figure 5a shows the effect of $I_{\mathrm{f}}$ on abnormal ventricular automatic activities constructed in the Tusscher-Noble-Noble-Panfilov model. In this case, the automaticity was elicited by the application of a depolarizing current following the augmentation of $I_{\mathrm{Ca}-\mathrm{L}}$ and $I_{\mathrm{Ks}}$ currents, which mimics the marginal area of myocardial infarction under sympathetic stimulation. As expected for the pacemaker current, implementation of $I_{\mathrm{f}}$ accelerated the rhythm because the current
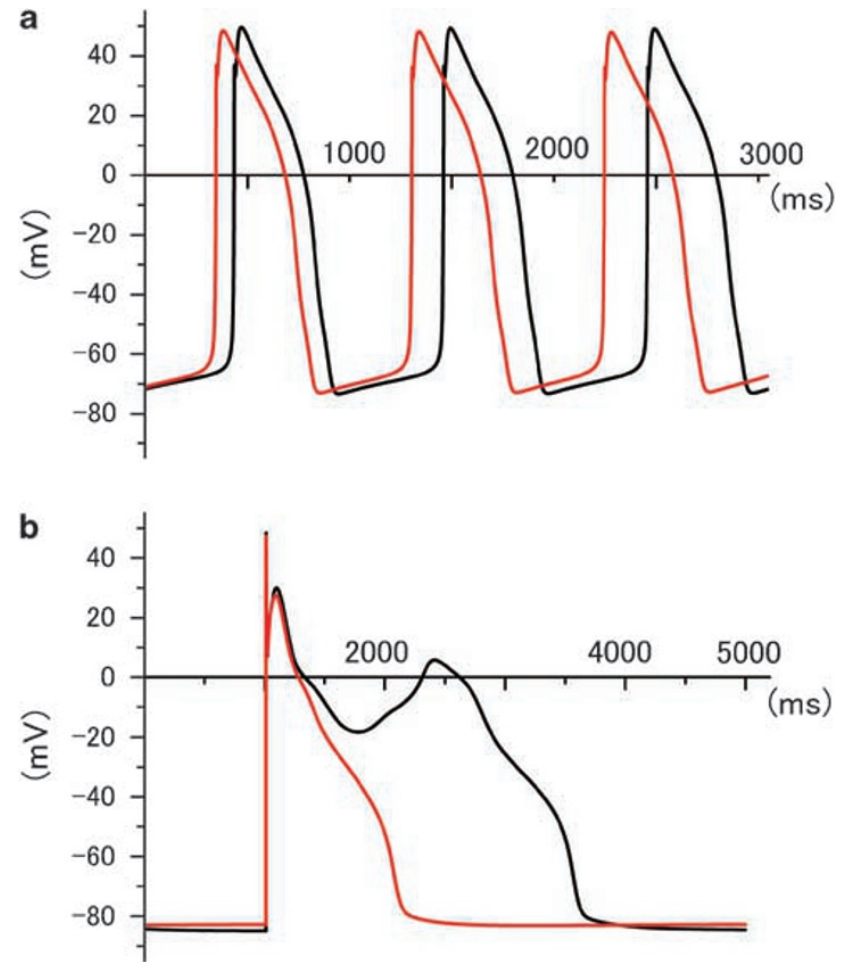

Figure 5 Effects of $I_{f}$ current on (a) ventricular abnormal automaticity, and (b) EAD in heart failure model. (a) Application of depolarizing injury current $\left(1.8 \mu \mathrm{A}_{\mu} \mathrm{F}^{-1}\right)$ into the TNNP model produced repetitive automatic activity. To simulate $\beta$-adrenergic stimulation observed in myocardial infarction, the amplitude of $I_{\mathrm{CaL}}$ and $I_{\mathrm{Ks}}$ were increased by factors of 4 and 2 , respectively. Incorporation of $l_{\mathrm{f}}$ into this situation accelerated the automaticity as shown by a red line. (b) The PB human ventricular action potential model was set to 'heart failure', characterized by long APD and slowed decay of $\mathrm{Ca}$ transient. ${ }^{25}$ Reduction of $I_{k r}$ in the PB heart failure model elicited EADs as shown by a black line. Incorporation of $I_{f}$ prevented EAD production (red line).

was inward, below the reversal potential. Figure 5b, on the other hand, shows the effect of plateau outward $I_{\mathrm{f}}$ current on the action potential repolarization. In this case, the Priebe-Beuckleman action potential model $^{25}$ was used, as it was mainly concerned with the modelling of cardiac failure. In the Priebe-Beuckleman 'heart failure' model, reduction in the $I_{\mathrm{Kr}}$ current produced early after depolarizations (EADs). ${ }^{25}$ As shown in Figure 5b, unusual APD prolongation owing to EAD was prevented by the inclusion of the $I_{\mathrm{f}}$ current.

\section{DISCUSSION}

In this study, we found several mutations in the channel genes from patients with ventricular arrhythmia, especially a novel HCN4 mutation in a symptomatic BrS patient, which caused abnormal splicing. In addition, we showed from the computer simulations that the $I_{\mathrm{f}}$ channel produced a background current contributing to the action potential repolarization of ventricular cardiomyocytes. The background current was generated by a mixed ion-selectivity for $\mathrm{Na}^{+}$ and $\mathrm{K}^{+}$, and incomplete closure for deactivation gate of $I_{\mathrm{f}}$ channels. This is the first report suggesting that mutation in HCN4 may render the bradycardia-induced ventricular arrhythmias.

Our study suggested that IVS2DS would partially affect the splicing. The presence of unaffected splicing may explain why SND was not observed in the patient. Although we did not prove the presence of truncated HCN4 channel in the patient, such channel should not be 
functional. When the abnormal splicing had occurred after the 4-bp insertion (at the abnormal donor site) and ended at the normal accepter site before exon 3, the inserted four nucleotides were included into mRNA, which was predicted to encode for a truncated channel of 447 residues containing non-relevant 44 amino-acid sequences at the C-terminal side. The predicted truncated channel should not form functional tetramers, and hence would be defective. It could be speculated that the abnormal splicing product might be unstable owing to the nonsense-mediated decay of mRNA. On the other hand, a cyclic nucleotide binding a domain-lacking mutation, P544fs, in HCN4 was associated with idiopathic SND, and the phenotype was a defective response of heart rate to exercise. ${ }^{2}$ The mutations IVS2DS and G553N ${ }^{1}$ were found in patients with frequent recurrence of ventricular tachycardia or syncope but were found neither in healthy controls nor in asymptomatic arrhythmia patients who had never experienced any life-threatening arrhythmia. These observations may provide an additional clue linking the HCN4 mutations and arrhythmogenesis. HCN4 mutations would affect channel property even if they were not responsible for any overt phenotypes, and exposing arrhythmic patients to slow heart rate was considered to put them into danger.

The $I_{\mathrm{f}}$ channels encoded by HCN2 and HCN4 in the heart are called as pacemaker channels, and expressed preferentially in cells with automaticity, thus playing a crucial role in impulse formation in the sinus node cells and cells in the specialized conduction system. However, it remains to be clarified whether and how the $I_{\mathrm{f}}$ channels would function in the working cardiomyocytes because of its ubiquitous expression in the heart. ${ }^{1,18}$ We incorporated the $I_{\mathrm{f}}$ current into three ventricular action potential models (the Tusscher-Noble-NoblePanfilov model for human ventricular cardiomyocytes, the PriebeBeuckelmann model for ventricular myocytes adopted for human heart failure and the Ruo-Rudy model that is widely used for ventricular cardiomyocytes) based on the kinetic parameters experimentally determined in this study (Figure 3 ) and on the current amplitude from the previous report using human ventricular cardiomyocytes. ${ }^{28}$ Our studies showed the inward $I_{\mathrm{f}}$ current during the diastole and the outward $I_{\mathrm{f}}$ current during the plateau of action potentials. At the physiological conditions, however, the effect of $I_{\mathrm{f}}$ on action potential was not prominent. The $I_{\mathrm{f}}$ inward current depolarized the resting potential a little, whereas the plateau outward current contributed to slight a abbreviation of APD (Figure 4). This was a common finding of $I_{\mathrm{f}}$ incorporation in all three simulation models tested in this study, although the effects on action potential parameters showed quantitative variations.

$I_{\mathrm{f}}$ repeats the cycle of activation at the diastole phase and deactivation during the action potential. When the action potential was elicited, the outward current amplitude during the early phase of repolarization was determined by the duration and the voltage of resting state, and also by the persistent component of the activation curve, $\min P_{0}$ (Figure 4). Therefore, the effects of $I_{\mathrm{f}}$ should be exaggerated during bradycardia, which may provide with long diastolic intervals. In other words, $I_{\mathrm{f}}$ may contribute to breaking down the excessive prolongation of APD when heart rates were decreased.

As the membrane conductance of ventricular cardiomyocytes is extremely low at the plateau phase, only a slight change in current amplitude may affect the membrane potential to determine APD. We showed here an HCN4 mutation that would reduce the $I_{\mathrm{f}}$ current owing to the possible formation of a truncated channel. Therefore, there might be circumstances where this type of HCN4 mutation contributed to arrhythmogenesis through the prolongation of APD, as it 'pushed off the safety catch'. Our simulation results in the Priebe-
Beuckelmann model (Figure 5) support this assumption. In fact, it was possible to produce situations where the presence of $I_{\mathrm{f}}$ current played critical roles to suppress EAD in all three models.

In conclusion, we identified a novel HCN4 mutation in 1 out of 33 (3\%) patients with BrS. The mutation was showed to cause splicing abnormality in transfected cells. Simulation studies suggested that the $I_{\mathrm{f}}$ currents produced by the HCN4 channel played a preventive role against the bradycardia-induced ventricular arrhythmia.

\section{ACKNOWLEDGEMENTS}

We thank Drs J Yamada, T Miura, M Kanamoto, T Tamura, N Miya, Y Horie, H Sakurada, S Ogawa, M Nishizaki, T Sakamoto, T Ueyama, K Hirao and J Nitta for their contributions in collecting blood samples and clinical records from the patients with BrS and LQTS. This study was supported in part by Grant-in-Aids from the Ministry of Education, Culture, Sports, Science and Technology, Japan and a research grant from the Ministry of Health, Labour and Welfare, Japan.

1 Ueda, K., Nakamura, K., Hayashi, T., Inagaki, N., Takahashi, M., Arimura, T. et al. Functional characterization of a trafficking-defective HCN4 mutation, D553N, associated with cardiac arrhythmia. J. Biol. Chem. 279, 27194-27198 (2004).

2 Schulze-Bahr, E., Neu, A., Friederich, P., Kaupp, UB., Breithardt, G., Pongs, O. et al. Pacemaker channel dysfunction in a patient with sinus node disease. J. Clin. Invest. 111, 1537-1545 (2003).

3 Wang, Q., Curran, M. E., Splawski, I., Burn, T. C., Millholland, J. M., VanRaay, T. J. et al. Positional cloning of a novel potassium channel gene: KVLQT1 mutations cause cardiac arrhythmias. Nat. Genet. 12, 17-23 (1996).

4 Curran, M. E., Splawski, I., Timothy, K. W., Vincent, G. M., Green, E. D. \& Keating, M. T. A molecular basis for cardiac arrhythmia: HERG mutations cause long QT syndrome. Cell 80, 795-803 (1995).

5 Wang, Q., Shen, J., Splawski, I., Atkinson, D., Li, Z., Robinson, J. L. et al. SCN5A mutations associated with an inherited cardiac arrhythmia, long QT syndrome. Cel/ 80, 805-811 (1995)

6 Splawski, I., Tristani-Firouzi, M., Lehmann, M. H., Sanguinetti, M. C. \& Keating, M. T. Mutations in the hminK gene cause long QT syndrome and suppress IKs function. Nat. Genet. 17, 338-340 (1996).

7 Abbott, G. W., Sesti, F., Splawski, I., Buck, M. E., Lehmann, M. H., Timothy, K. W. et al. MiRP1 forms $\mathrm{IKr}$ potassium channels with HERG and is associated with cardiac arrhythmia. Cell 97, 175-187 (1997).

8 Tristani-Firouzi, M., Jensen, J. L., Donaldson, M. R., Sansone, V., Meola, G., Hahn, A. et al. Functional and clinical characterization of KCNJ2 mutations associated with LQT7 (Andersen syndrome). J. Clin. Invest. 110, 381-388 (2002).

9 Splawski, I., Timothy, K. W., Sharpe, L. M., Decher, N., Kumar, P., Bloise, R. et al. $\mathrm{Ca}(\mathrm{V}) 1.2$ calcium channel dysfunction causes a multisystem disorder including arrhythmia and autism. Cell 119, 19-31 (2004).

10 Brugada, R., Hong, K., Dumaine, R., Cordeiro, J., Gaita, F., Borggrefe, M. et al. Sudden death associated with short-QT syndrome linked to mutations in HERG. Circulation 109, 30-35 (2004).

11 Bellocq, C., van Ginneken, A. C., Bezzina, C. R., Alders, M., Escande, D., Mannens, M. $M$. et al. Mutation in the KCNQ1 gene leading to the short QT-interval syndrome. Circulation 109, 2394-2397 (2004)

12 Chen, Y. H., Xu, S. J., Bendahhou, S., Wang, X. L., Wang, Y., Xu, W. Y. et al. KCNQ1 gain-of-function mutation in familial atrial fibrillation. Science 299, 251-254 (2003).

13 Yang, Y., Xia, M., Jin, Q., Bendahhou, S., Shi, J., Chen, Y. et al. Identification of a KCNE2 gain-of-function mutation in patients with familial atrial fibrillation. Am. J. Hum. Genet. 75, 899-905 (2004).

14 Chen, Q., Kirsch, G. E., Zhang, D., Brugada, R., Brugada, J., Brugada, P. et al. Genetic basis and molecular mechanism for idiopathic ventricular fibrillation. Nature 392, 293-296 (1998).

15 Schott, J. J., Alshinawi, C., Kyndt, F., Probst, V., Hoorntje, T. M., Hulsbeek, M. et al. Cardiac conduction defects associate with mutations in SCN5A. Nat. Genet. 23, 20-21 (1999).

16 Priori, S. G., Napolitano, C., Tiso, N., Memmi, M., Vignati, G., Bloise, R. et al. Mutations in the cardiac ryanodine receptor gene (hRyR2) underlie catecholaminergic polymorphic ventricular tachycardia. Circulation 103, 196-200 (2001).

17 Marban, E. Cardiac channelopathies. Nature 415, 213-218 (2002).

18 Moosmang, S., Stieber, J., Zong, X., Biel, M., Hofmann, F. \& Ludwig, A. Cellular expression and functional characterization of four hyperpolarization-activated pacemaker channels in cardiac and neuronal tissues. Eur. J. Biochem. 268, 1646-1652 (2001).

19 Westenskow, P., Splawski, I., Timothy, K. W., Keating, M. T. \& Sanguinetti, M. C. Compound mutations: a common cause of severe long-QT syndrome. Circulation 109, 1834-1841 (2004). 
20 Nishi, H., Kimura, A., Harada, H., Toshima, H. \& Sasazuki, T. Novel missense mutation in cardiac beta myosin heavy chain gene found in a Japanese patient with hypertrophic cardiomyopathy. Biochem. Biophys. Res. Commun. 188, 379-387 (1992).

21 Wang, Q., Li, Z., Shen, J. \& Keating, M. T. Genomic organization of the human SCN5A gene encoding the cardiac sodium channel. Genomics 34, 9-16 (1996).

22 Splawski, I., Shen, J., Timothy, K. W., Vincent, G. M., Lehmann, M. H. \& Keating, M. T. Genomic structure of three long QT syndrome genes: KVLQT1, HERG, and KCNE1. Genomics 51, 86-97 (1998).

23 Plaster, N. M., Tawil, R., Tristani-Firouzi, M., Canun, S., Bendahhou, S., Tsunoda, A. et al. Mutations in Kir2.1 cause the developmental and episodic electrical phenotypes of Andersen's syndrome. Cell 105, 511-519 (2001).

24 Chen, J., Mitcheson, J. S., Tristani-Firouzi, M., Lin, M. \& Sanguinetti, M. C. The S4-S5 linker couples voltage sensing and activation of pacemaker channels. Proc. Natl. Acad. Sci. 98, 11277-11282 (2001).
25 Priebe, L. \& Beuckelmann, D. J. Simulation study of cellular electric properties in heart failure. Circ. Res. 82, 1206-1223 (1998).

26 ten Tusscher, K. H., Noble, D., Noble, P. J. \& Panfilov, A. V. A model for human ventricular tissue. Am. J. Physiol. Heart Circ. Physiol. 286, H1573-H1589 (2004).

27 Luo, C. H. \& Rudy, Y. A dynamic model of the cardiac ventricular action potential: I. Simulations of ionic currents and concentration changes. Circ. Res. 74, 1071-1096 (1994).

28 Cerbai, E., Sartiani, L., de Paoli, P., Pino, R., Maccherini, M., Bizzarri, F. et al. The properties of the pacemaker current I(F)in human ventricular myocytes are modulated by cardiac disease. J. Mol. Cell. Cardiol. 33, 441-448 (2001).

29 Kaupp, U. B. \& Seifert, R. Molecular diversity of pacemaker ion channels. Annu. Rev. Physiol. 63, 235-257 (2001).

30 Brugada, P., Geelen, P., Brugada, R., Mont, L. \& Brugada, J. Prognostic value of electrophysiologic investigations in Brugada syndrome. J. Cardiovasc. Electrophysiol. 12, 1004-1007 (2001). 